\title{
Factors Related to Outcomes of Fecal Microbiota Transplantation in Patients with Clostridioides difficile Infection
}

\author{
Hyuk Yoon ${ }^{1}$, Hyun Ik Shim¹, Mijin Seol², Cheol Min Shin ${ }^{1}$, Young Soo Park¹, Nayoung Kim ${ }^{1,3}$, and Dong Ho Lee ${ }^{1,2,3}$ \\ ${ }^{1}$ Department of Internal Medicine, Seoul National University Bundang Hospital, ${ }^{2}$ R\&D Center, BioBankHealing Inc., Seongnam, and \\ ${ }^{3}$ Department of Internal Medicine and Liver Research Institute, Seoul National University College of Medicine, Seoul, Korea
}

See editorial on page 1.

\section{Article Info}

Received April 23, 2020

Revised June 8, 2020

Accepted June 28, 2020

Published online September 1, 2020

\section{Corresponding Author}

Dong Ho Lee

ORCID https://orcid.org/0000-0002-6376-410X

E-mail dhljohn@yahoo.co.kr
Background/Aims: The aim of this study was to evaluate factors related to outcomes of fecal microbiota transplantation (FMT) in patients with Clostridioides difficile infection (CDI) and viability of frozen stock for FMT.

Methods: Clinical data of patients who had received FMT for CDI were prospectively collected. Next-generation 16S rRNA gene sequencing of bacteria was performed from donors' and recipients' stool. Colony-forming units (CFUs) of cultures from frozen stock solutions for FMT were measured at $0,4,8,12,24,48$ weeks after preparation of the solutions.

Results: In total, 25 FMT procedures were performed in 20 cases (14 fresh and 11 frozen FMT). Forty-five percent of cases involved fulminant CDI. The overall success rate was $55 \%$ after the 1st FMT and $75 \%$ after the 2nd FMT. The success rate was significantly higher in partially treated CDI than in refractory CDI $(100 \%$ vs $71.4 \% ; p=0.001)$. In successful cases only, the decrease in alpha-diversity in the recipient stool microbiomes was recovered after FMT to a level similar to that in donor stools. There was a significant difference in the microbiome composition in pre-FMT recipients' stool between successful and failed cases $(p=0.001)$. The CFUs of frozen solution for FMT did not decrease for 48 weeks in both aerobic and anaerobic cultures.

Conclusions: FMT is highly effective in partially treated CDI but not in refractory CDI. The microbiome differs between failed and successful cases. Frozen stock for FMT is viable up to 48 weeks. (Gut Liver 2021;15:61-69)

Key Words: Clostridioides difficile infection; Clostridium difficile infection; Fecal microbiota transplantation

\section{INTRODUCTION}

There have been many studies on the effect of fecal microbiota transplantation (FMT) in Clostridioides (formerly Clostridium) difficile infection (CDI). FMT is generally accepted as a very effective treatment in recurrent $\mathrm{CDI},{ }^{1,2}$ and is considered as a treatment option for refractory CDI. In recent meta-analysis, the overall clinical resolution rate of recurrent and refractory CDI after FMT was $92 \%{ }^{3}$ However, the indication and protocol of FMT vary among studies and guidelines. In addition, there are multiple factors that can influence the outcome of FMT.

In clinical trials, CDI has been commonly classified as recurrent or refractory. However, in practice, there are patients whose disease fits a pattern intermediate between typical recurrent or refractory CDI. ${ }^{4} \mathrm{CDI}$-related symptoms usually improve after 5-7 days and 3-5 days of metronidazole and vancomycin treatment, respectively. ${ }^{5}$ However, some patients initially respond to antibiotics, but do not show complete resolution of the symptoms. This phenomenon is more common in severe CDI, in which the bacterial load is high. In these patients, it can be expected that the risk of worsening of symptoms is very high when antibiotics are stopped after the recommended period. However, there has been no study which specifically focused on the effect of FMT in patients with partially treated CDI.

Therefore, we set out to evaluate the factors related 
to the outcome of FMT in patients with CDI, including patients who showed partial response to antibiotics. In addition, because frozen FMT has been becoming more and more popular in Korea, we demonstrated long viability of frozen stock for FMT.

\section{MATERIALS AND METHODS}

\section{Subjects}

Consequent patients with CDI who decided to receive FMT were prospectively enrolled. Inclusion criteria were as follows: (1) patients with confirmed CDI who were at least 19 years old; (2) CDI was not completely resolved after appropriate antibiotics treatment; (3) patients and caregivers did not want total colectomy, or patients were not eligible for surgery due to comorbidities. For patients with partially treated primary CDI, FMT was suggested based on the fact that the evidence for adjunctive or alternative treatments such as rifaximin and probiotics is very low, ${ }^{1}$ and there have been some positive reports regarding FMT for primary CDI. ${ }^{6,7}$ Exclusion criteria were as follows: (1) patients for whom an appropriate stool donor could not be found; (2) moderate to severe immunocompromised patients; or (3) non-compensated liver cirrhosis. All subjects provided informed consent and the study was approved by the Institutional Review Board of the Seoul National University Bundang Hospital (IRB number: B-1507-305-006).

\section{Definitions}

CDI was defined when a patient showed compatible symptoms and signs (fever, abdominal pain, and diarrhea or ileus), and the toxin assay for $C$. difficile was positive or typical features of pseudomembranous colitis were found in colonoscopy. ${ }^{1,8}$ Because endoscopy is not prerequisite for diagnosis of CDI, and most patients were in poor medical condition, it was performed before FMT only in patients in whom diagnosis was uncertain by symptoms and the toxin assay. In remaining patients, colon was evaluated during FMT procedure. Severe complicated (fulminant) CDI was defined according to American College of Gastroenterology guidelines. ${ }^{5}$ Response to antibiotics was evaluated after 3 to 5 days of treatment. In patients showing a partial response, the final decision was re-evaluated on 7 days. Patients who did not show complete resolution of CDI were classified as partially treated or refractory CDI when they were referred to a gastroenterologist to discuss performing FMT procedures. Partially treated CDI was defined when a patient showed improvement, but his/her condition was stationary, without complete resolution of CDI. Refractory CDI was defined when CDI was not improved or even ag- gravated after appropriate antibiotic treatment. The decision was made based on the combination of symptoms/ signs (fever, abdominal pain/tenderness, diarrhea/ileus, blood pressure, and mental status) and laboratory results (white blood cells and C-reactive protein). Other possible causes such as antibiotic-associated diarrhea and irritable bowel syndrome were carefully examined. The primary endpoint was treatment success rate after receiving up to two FMTs without the need of antibiotics. Because a uniformly agreed definition is currently lacking, this was defined as resolution of the symptoms on clinical grounds on day 5 to 7 after FMT according to a consensus. ${ }^{9}$ In cases where the decision was difficult, follow-up endoscopy was adjunctively performed. The secondary endpoint was absence of symptoms within 90 days after FMT.

\section{FMT procedures}

We screened potential donors in personal interviews. We excluded subjects who had chronic diseases or subjects who had taken any antibiotics within 3 months. For potential donors, infectious diseases were excluded by serologic testing and stool tests. In recipients, antibiotics were stopped 12 hours before the FMT procedure and bowel preparation was performed. For fresh FMT, 50 to $100 \mathrm{~g}$ of donor stool was collected immediately after defecation on the day of the procedure. It was diluted in normal saline in the ratio of 1:3. Then, it was homogenized in a blender; large particles were removed using a filter. The final solution was evenly sprayed onto the entire surface of the recipient's colon. In patients for whom the risk of colon perforation was high, FMT solution was sprayed in the third part of the duodenum using esophagogastroduodenoscopy. ${ }^{10}$ When fresh donor stool was not available, frozen FMT was performed. Glycerol added solution was frozen at $-80^{\circ} \mathrm{C}$. The frozen solution was thawed as described in a previous study, ${ }^{11}$ and it was transplanted using the same methods as those for fresh FMT. For non-responders, 2nd FMT was performed after 1 week. All endoscopic procedures were performed by one gastroenterologist (H.Y.).

\section{Collection of stool and clinical data}

Stool samples of recipients were collected the day before and 1 week after the FMT procedure. We compared the stool microbiome among donors, pre-FMT recipients, and post-FMT recipients in successful cases and failed cases, respectively. In addition, we compared the pre-FMT stool microbiome between successful and failed cases. Clinical data were collected using a standardized case report form. To evaluate the long-term effect of FMT, we followed up the recipients of successful cases. 


\section{Microbiome analysis}

Total DNA was extracted from stool samples, and polymerase chain reaction amplification for V3-V4 regions of the 16S rRNA gene was performed. Sequencing was carried out using an Illumina MiSeq Sequencing system (IIlumina, San Diego, CA, USA). Basic microbiome analyses were conducted according to previously described procedures. ${ }^{12-15}$ For analysis of the alpha-diversity, the Shannon diversity index at a $3 \%$ distance was calculated. For analysis of the beta-diversity, the overall phylogenetic distance between communities was estimated and visualized using Jensen-Shannon-based principal coordinates analysis; permutational multivariate analysis of variance was used to evaluate the set-difference between groups.

\section{Culture and colony counting of the solution for frozen FMT}

To verify the microbial viability of the solution for frozen FMT, we counted colony-forming units (CFUs) of cultures from the frozen stocks at various time points. Before freezing the FMT solution, $1 \mathrm{~mL}$ of each solution was collected and refrigerated at $-80^{\circ} \mathrm{C}$. The viable stocks were thawed and serially diluted, and they were smeared on Brain Heart Infusion agar and Gifu Anaerobic Medium agar plates. The plates were incubated at $37^{\circ} \mathrm{C}$ in aerobic and anaerobic conditions for 48 hours. For the anaerobic condition, the plates were placed in a jar from which oxygen was removed using the AnaeroPack system (Mitsubishi Gas Chemical, Tokyo, Japan), and cultures were incubated in an anaerobic chamber. The CFU was calculated at $0,4,8$, 12, 24, 48 weeks.

\section{Statistical analysis}

STATA version 16.0 was used for statistical analysis of the clinical data. Continuous variables were analyzed using the Mann-Whitney $U$ test and Friedman test, because the data were not normally distributed. The Fisher exact test was used to analyze categorical variables. Results were considered statistically significant when p-values were less than 0.05 .

\section{RESULTS}

\section{Baseline characteristics of the recipients}

Between March 2016 and October 2018, 25 FMT procedures in total were performed in 20 cases (14 fresh and 11 frozen FMT). Baseline characteristics of the recipients are shown in Table 1 . Ninety percent of the patients was more than 65 years of age; the median recipient age was 78.5 years. Seventy percent of the patients exhibited Charlson comorbidity index $\geq 3$. Forty-five percent of cases was fulminant CDI; $20 \%$ of the patients were intubated. In 11 patients (55\%), index CDI was the first episode; the median number of episodes was three in recurrent cases.

\section{Characteristics and outcomes of FMT procedures}

Table 2 shows the characteristics of FMT procedures. The median donor age was 46.5 years, and most donors (80\%) were a family member of a recipient. One patient underwent FMT using a mixed solution from two universal donors. FMT was performed 11 days (median) after the diagnosis of CDI. Antegrade and retrograde approach was $44 \%$ and 56\%, respectively. Second FMT was performed in $20 \%$ of the patients. There were no adverse events related to the FMT procedure.

The overall success rate was 55\% after 1st FMT, and

Table 1. Baseline Characteristics of the Recipients

\begin{tabular}{|c|c|}
\hline Variable & Value \\
\hline Male sex & $10(50)$ \\
\hline Age, yr & $78.5(41-91)$ \\
\hline Charlson comorbidity index & $3(0-8)$ \\
\hline \multicolumn{2}{|l|}{ Location of patient } \\
\hline General ward & $13(65)$ \\
\hline Intensive care unit & $6(30)$ \\
\hline Outpatient department & $1(5)$ \\
\hline Intubated & $4(20)$ \\
\hline On hemodialysis & $4(20)$ \\
\hline \multicolumn{2}{|l|}{ Index infection before CDI } \\
\hline Urinary tract infection & $8(40)$ \\
\hline Pneumonia & $4(20)$ \\
\hline Others* & $2(10)$ \\
\hline Prophylactic use of antibiotics & $4(20)$ \\
\hline None & $2(10)$ \\
\hline \multicolumn{2}{|l|}{ Medications used to treat index $\mathrm{CDI}^{+}$} \\
\hline Metronidazole, oral & $3(15)$ \\
\hline Metronidazole, intravenous & 15 (75) \\
\hline Vancomycin, oral & $18(90)$ \\
\hline Vancomycin, enema & $10(50)$ \\
\hline \multicolumn{2}{|l|}{ Indication of FMT } \\
\hline Partial response to antibiotics & $13(65)$ \\
\hline Refractory to antibiotics & 7 (35) \\
\hline Event No. of CDI in recurrent cases & $3(2-5)$ \\
\hline Endoscopic finding suitable for PMC & $18(90)$ \\
\hline Stool frequency & $2.5(0-14)$ \\
\hline Body temperature $37.8^{\circ} \mathrm{C}$ & $4(20)$ \\
\hline White blood cells, $\times 10^{3} / \mu \mathrm{L}$ & $9.0(4.1-17.4)$ \\
\hline C-reactive protein, $\mathrm{mg} / \mathrm{dL}$ & $3.5(0.4-18.3)$ \\
\hline Albumin, g/dL & $2.7(1.9-3.9)$ \\
\hline Creatinine, mg/dL & $0.6(0.4-6.5)$ \\
\hline
\end{tabular}

Data are presented as number (\%) or median (range).

$\mathrm{CDI}$, Clostridioides difficile infection; FMT, fecal microbiota transplantation; PMC, pseudomembranous colitis.

*Others: 2, Tb peritonitis, cholangitis; ${ }^{\dagger}$ All medications used to treat $\mathrm{CDI}$ were independently calculated. 
Table 2. Characteristics of FMT Procedures

\begin{tabular}{lc}
\hline \multicolumn{1}{c}{ Variable } & Value \\
\hline Sex of donor (male) & $12(60)$ \\
Age of donor, yr & $46.5(20-66)$ \\
Relationship of donor with recipient & \\
Family member & $16(80)$ \\
Acquaintance & $3(15)$ \\
Universal multi-donor & $1(5)$ \\
Interval between diagnosis of CDI and 1st FMT, day & $11(2-35)$ \\
Type of FMT & \\
Fresh & $13(65)$ \\
Frozen & $7(35)$ \\
Route of FMT* & \\
Antegrade & $11(44)$ \\
Retrograde & $14(56)$ \\
Volume of donor stool, $\mathrm{g}^{*}$ & $100(17-350)$ \\
2nd FMT & $5(25)$ \\
\hline
\end{tabular}

Data are presented as number (\%) or median (range).

FMT, fecal microbiota transplantation; CDI, Clostridioides difficile infection.

${ }^{*}$ Calculated based on 25 procedures in 20 recipients; ${ }^{\dagger}$ The second FMT was successful in four recipients.

75\% after 2nd FMT. A comparison between successful and failed cases is shown in Table 3. The success rate was significantly higher in partially treated CDI than in refractory CDI $(100 \%$ vs $71.4 \% ; p=0.001)$. The interval between diagnosis of CDI and 1st FMT procedure was significantly shorter in failed cases than in successful cases (median, 8 days vs 15 days; $\mathrm{p}=0.044$ ). Other factors related to the FMT procedures, such as route (antegrade vs retrograde) and method (fresh vs frozen), did not affect the outcome of FMT.

Among five patients who failed FMT, one patient was given oral vancomycin due to continuous abdominal pain, after which the symptom was resolved. Another patient underwent total colectomy and recovered. The other three patients expired. The causes of death were septic shock, progression of pulmonary thromboembolism, and recurred stroke. The median follow-up period of the 15 successful cases was 8 months. CDI recurred in one patient (6.7\%) within 90 days. Two patients expired due to reasons irrelevant to CDI (progression of esophageal cancer and congestive heart failure). There was no recurrence of CDI in the remaining patients.

\section{Composition of the stool microbiome and follow- up culture of frozen solution for FMT}

A total of 55 stool samples were analyzed; two and three samples were missing in the collection of donors and postFMT recipients, respectively. In successful cases, the proportion of Firmicutes plus Bacteroidetes was 84.5\%, 43.3\%, and $56.4 \%$ in donor, pre-FMT recipient, and post-FMT
Table 3. Comparison of Recipient and Procedural Characteristics According to the Outcome of FMT

\begin{tabular}{|c|c|c|c|}
\hline Variable & Success & Failure & $p$-value \\
\hline Male sex & $7(46.7)$ & $3(60.0)$ & 1.0 \\
\hline Old age ( $\geq 65 \mathrm{yr}$ ) & 13 (86.7) & $5(100)$ & 1.0 \\
\hline Charlson comorbidity index $\geq 3$ & $9(60.0)$ & $5(100)$ & 0.260 \\
\hline Fulminant CDI & 5 (33.3) & $4(80.0)$ & 0.127 \\
\hline Indication of FMT & & & 0.001 \\
\hline Partial response to antibiotics & 13 (86.7) & 0 & \\
\hline Refractory to antibiotics & $2(13.3)$ & $5(100)$ & \\
\hline Interval between CDI and FMT, median, day & 15 & 8 & 0.044 \\
\hline Type of FMT & & & 1.0 \\
\hline Fresh & 10 (66.7) & $3(60.0)$ & \\
\hline Frozen & $5(33.3)$ & $2(40.0)$ & \\
\hline Route of FMT & & & 0.617 \\
\hline Antegrade & $6(40.0)$ & $3(60.0)$ & \\
\hline Retrograde & $9(60.0)$ & $2(40.0)$ & \\
\hline Age of donor, mean, yr & 45 & 47 & 0.793 \\
\hline Volume of donor stool $\geq 100 \mathrm{~g}$ & 10 (66.7) & $3(60.0)$ & 1.0 \\
\hline
\end{tabular}

Data are presented as number (\%).

FMT, fecal microbiota transplantation; CDI, Clostridioides difficile infection.

recipient stools, respectively (Fig. 1A). The proportion of Proteobacteria plus Fusobacteria was 7.6\%, 55.4\%, and $37.6 \%$ in donor, pre-FMT recipient, and post-FMT recipient stools, respectively. The decrease in alpha-diversity in the recipient stool microbiomes was recovered after FMT to a level similar to that in donor stools (Fig. 1B). There was a significant difference in the microbiome composition among the three groups $(\mathrm{p}=0.001)$. In pair-wise comparisons, each group was significantly different from the other groups (all three $\mathrm{p}<0.05$ ). Principal coordinates analysis suggested that the microbiome composition of post-FMT recipients was positioned between those of preFMT recipients and donors (Fig. 1C). In failed cases, the proportion of Firmicutes plus Bacteroidetes was $89.6 \%$, $33.0 \%$, and $37.6 \%$ in donor, pre-FMT recipient, and postFMT recipient stools, respectively (Fig. 2A). The proportion of Proteobacteria plus Fusobacteria was $<1 \%, 66.0 \%$, and $60.0 \%$ in donor, pre-FMT recipient, and post-FMT recipient stools, respectively. The stool microbiomes of donors were significantly different from those of pre- and post-FMT recipients in both alpha- and beta-diversity, but there was no difference between pre- and post-FMT recipients (Fig. 2B and C). We identified a significant difference in the microbiome composition of pre-FMT stool between successful and failed cases $(\mathrm{p}=0.001)$. There was no difference in the microbiome composition of donor stools between successful and failed cases $(\mathrm{p}=1.0)$.

CFUs of viable stocks from stools used for frozen FMT in aerobic and anaerobic cultures are shown in Fig. 3, respectively. The CFUs of viable stocks did not decrease for 

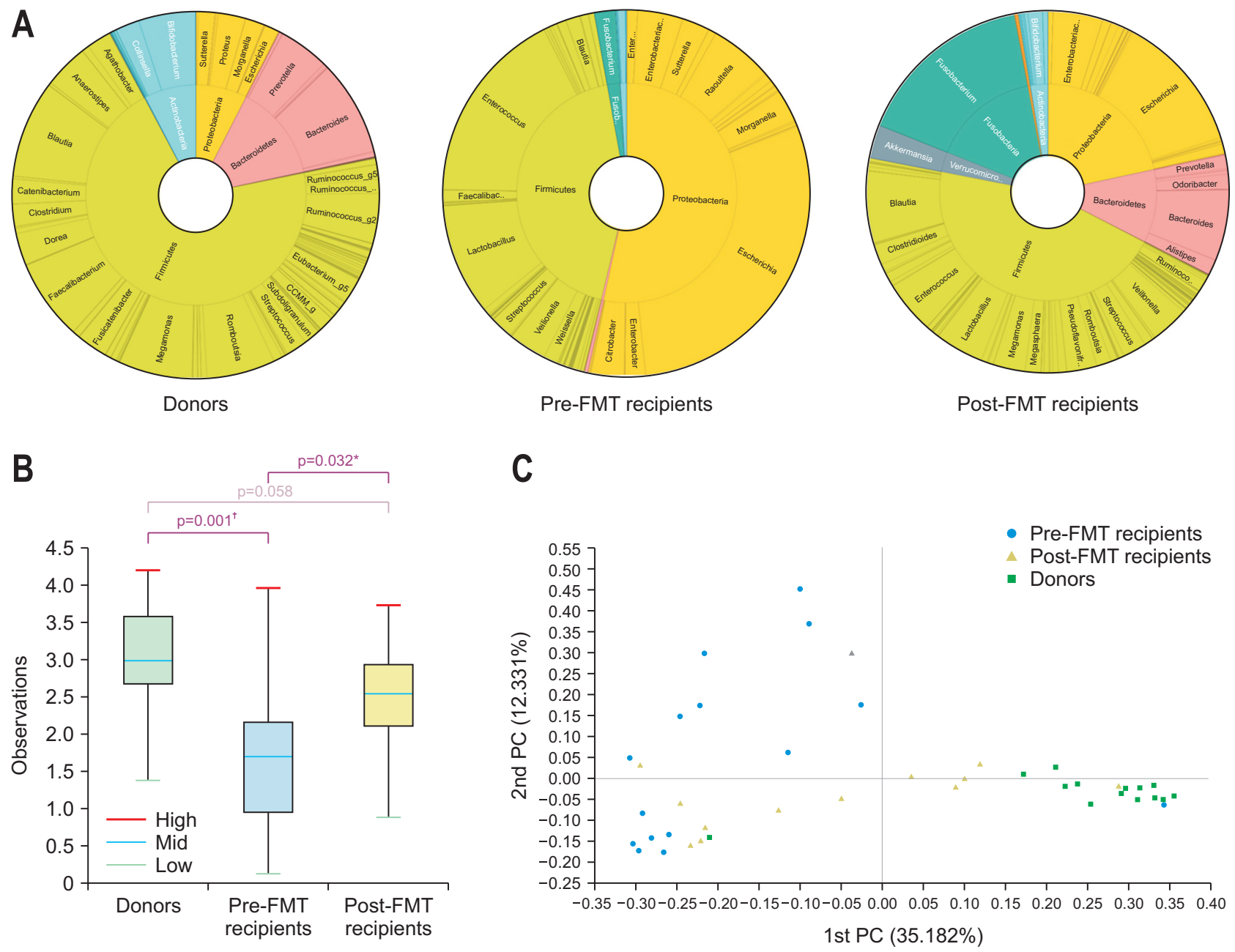

Fig. 1. (A) Taxonomic composition of the stool microbiome among donors, pre-fecal microbiota transplantation (pre-FMT) recipients, and post-FMT recipients in successful cases; double pie chart (inner circle: phylum level, outer circle: genus level). (B) Shannon index of the stool microbiome among donors, pre-FMT recipients, and post-FMT recipients in successful cases. (C) Jensen-Shannon-based principal coordinate (PC) analysis of the stool microbiome among donors, pre-FMT recipients, and post-FMT recipients in successful cases (genus level). ${ }^{*} p<0.05,{ }^{\dagger} p<0.001$.

48 weeks in both aerobic and anaerobic cultures $(\mathrm{p}=0.929$ and $\mathrm{p}=0.466$, respectively).

\section{DISCUSSION}

The success rate of FMT in the present study, 55\% after 1st FMT and 75\% after 2nd FMT, seems to be disappointing compared to that in previous studies. ${ }^{3}$ However, patients with partially treated CDI showed excellent responses to FMT, regardless of other factors. The success rate in these patients was $100 \%$. Previous studies have performed FMT in patients who showed recurrent symptoms during or after vancomycin tapering therapy ${ }^{16,17}$ In contrast, the patients with partially treated CDI in our study received FMT because they did not reach complete resolution of symptoms.
The most appropriate timing for FMT following antibiotic treatment in CDI is uncertain. Guidelines simply recommend that patients with recurrent CDI should be treated with vancomycin for at least 3 days before FMT. ${ }^{4,9}$ In some clinical trials, FMT was performed after 3 to 5 days of antibiotics. ${ }^{18,19}$ However, in daily practice, especially in institutions without a stool bank, it is very difficult to perform fresh FMT in this early phase of disease. It takes several days to recruit volunteers, screen potential donors, and collect donor stool. Often, it is impossible to obtain fresh stool on the day of scheduled FMT. In some cases, treatment is started with oral metronidazole, which is replaced with oral vancomycin when there is no response in several days. Other studies have performed FMT after resolution of the symptoms following a full course of antibiotics. ${ }^{17,20}$ However, in some patients, symptoms are not completely resolved after appropriate antibiotic treatment. 

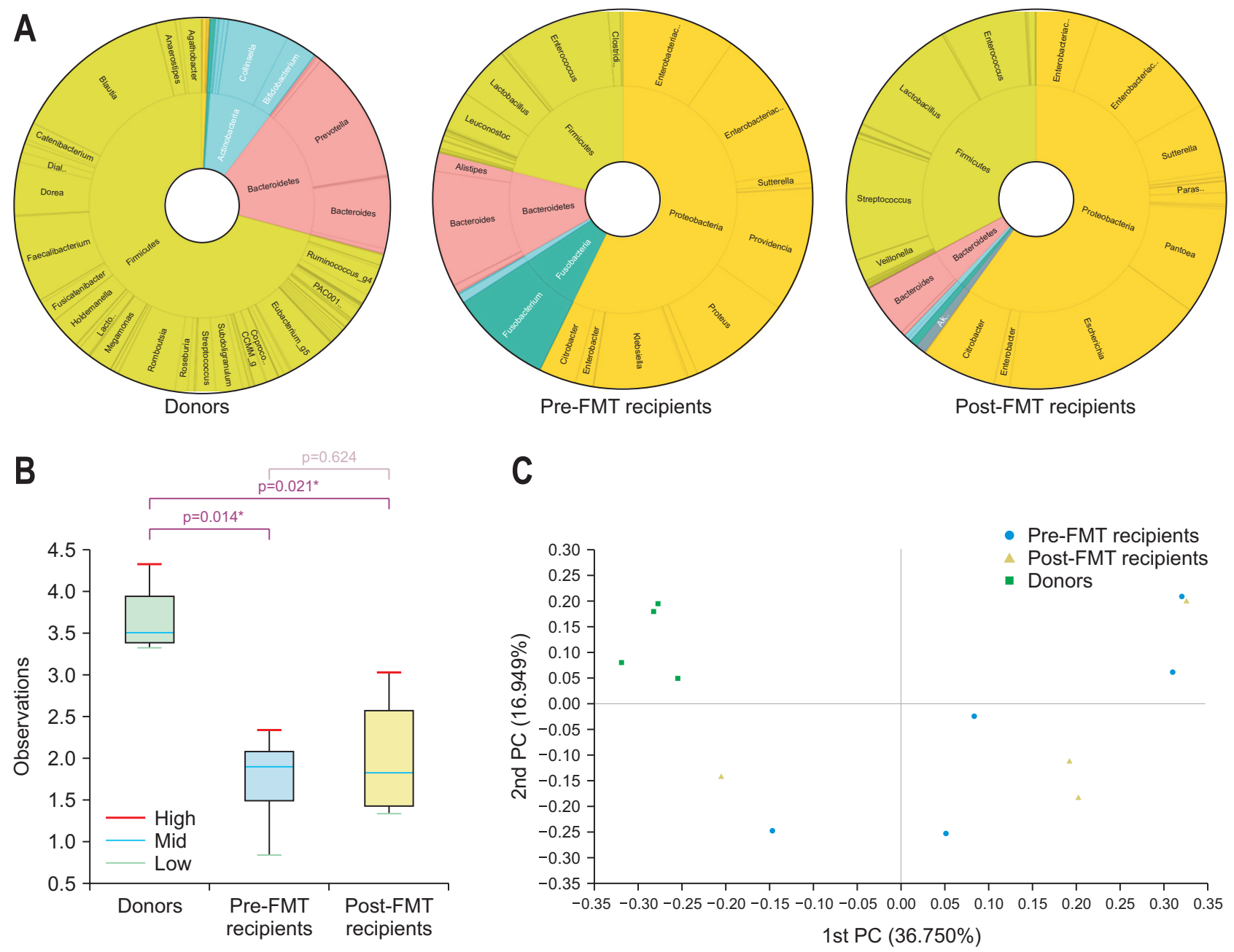

Fig. 2. (A) Taxonomic composition of the stool microbiome among donors, pre-fecal microbiota transplantation (pre-FMT) recipients, and post-FMT recipients in failed cases; double pie chart (inner circle: phylum level, outer circle: genus level). (B) Shannon index of the stool microbiome among donors, pre-FMT recipients, and post-FMT recipients in failed cases. (C) Jensen-Shannon-based principal coordinate (PC) analysis of the stool microbiome among donors, pre-FMT recipients, and post-FMT recipients in failed cases (genus level). * $p<0.05$.
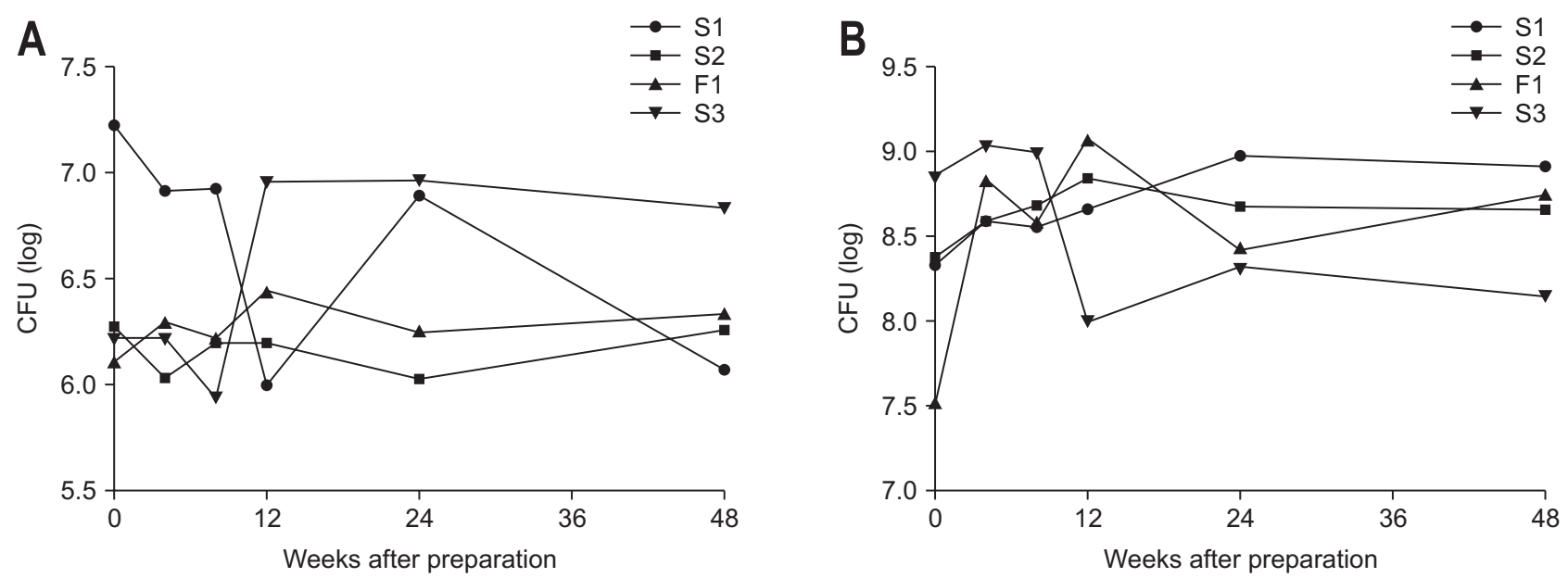

Fig. 3. Colony-forming units (CFUs) of viable stocks of frozen solution for microbiota transplantation in (A) aerobic culture and (B) anaerobic culture. $\mathrm{S}$, successful case; $F$, failed case. 
The results of this study suggest that FMT is a very good option for partially treated CDI patients.

The patients enrolled in this study had many disadvantages to achieve a successful outcome of FMT. As for recipient-related factors, the patients were advanced age (median age, 78.5 years) and serum albumin levels were very low (median, $2.7 \mathrm{~g} / \mathrm{dL}$ ). These are well-known predictive factors for poor outcome of FMT. ${ }^{21}$ The median value of the Charlson comorbidity index was three. The outcome of FMT in patients with multiple comorbidities is poor. ${ }^{20}$ Moreover, the burden of C. difficile was heavy; $45 \%$ was fulminant CDI and pseudomembranous colitis was found in the endoscopy in $90 \%$ of patients. Although the success rate of FMT in fulminant CDI $(55.6 \%, 5 / 9)$ was lower than those from previous studies ( $66 \%$ to $88 \%$ ), ${ }^{22,23}$ the success rate in patients with fulminant CDI which showed partial response to antibiotics was $100 \%(4 / 4)$. Our study suggests that despite other unfavorable factors, the outcome of FMT is very good in patients with partially treated CDI. Notably, in patients with fulminant CDI who were intubated, when the patients showed partial response to antibiotics, FMT was performed in the late stage and resulted in a good outcome.

In contrast, donor- or FMT procedure-related factors did not affect the outcome of FMT. As suggested in a previous study, ${ }^{4}$ sex, age, and relationship of the donor were not important factors. Comparison of the donor stool microbiomes between successful and failed cases supported this. Procedure-related factors were also not crucial. Except the indication of FMT, the only other factor that differed between successful and failed cases was the time interval between diagnosis of CDI and the FMT procedure. As multivariate analysis was not appropriate because of the small sample size, we instead evaluated the correlation between this factor and the indication of FMT. The interval between diagnosis of CDI and FMT procedure in partially treated CDI was significantly longer than that in refractory CDI (median, 17 days vs 8 days; $\mathrm{p}=0.010$ ). To conclude that FMT failure was due to the antibiotic treatment duration being less than 2 weeks is clinically irrational, and it is not supported by previous studies. Therefore, we reason that the interval between diagnosis of CDI and FMT procedure acted as confounder.

For refractory CDI that did not show any response to 1-week antibiotic treatment, the outcome of FMT was poor. This result supports that FMT can be considered in refractory CDI, but it does not provide a certain solution. The outcome of failed cases was grave; $60 \%$ of the patients expired. We should consider that the patients enrolled were generally a very high-risk population. Nevertheless, given these grave results, we suggest discussing other op- tions, such as surgery or new antibiotics (e.g., fidaxomicin) in refractory CDI. ${ }^{1,24}$ In contrast, CDI recurred in only $6.7 \%$ of the successful cases. This suggests that irrespective of the indication of FMT, if it succeeds to resolution of CDI, the effect lasts for a certain period.

In successful cases, the decrease in alpha-diversity in recipient stool microbiomes was recovered after FMT to a level similar to that in the donor stools. The proportion of Firmicutes and Bacteroidetes, which are the main two phyla in the healthy human gut, increased after FMT. In contrast, the proportion of Proteobacteria and Fusobacteria, which are commonly dominant in unhealthy colonic conditions, decreased. The microbial composition of recipient stools was shifted towards that of donor stools that after FMT. These phenomena were not observed in failed cases. These results are in good agreement with those in previous studies. ${ }^{25,26}$ In addition, there was a significant difference in the microbiome composition of pre-FMT stool between successful and failed cases. This implies that the composition of the stool microbiome might predict the outcome of FMT in patients with CDI. Further studies are required to evaluate this possibility.

A guideline recommends that frozen solution for FMT can be used for up to 6 months after preparation, based on a study that reported that viable aerobic and anaerobic bacterial counts did not decrease for 6 months in frozen stool. ${ }^{27}$ However, some institutes use longer storage periods of up to 2 years. ${ }^{28}$ In our study, outcomes did not differ when using fresh or frozen FMT. The viability of the frozen solution in both aerobe and anaerobe cultures did not decrease for up to 48 weeks after preparation of the solution. These results provide evidence that frozen solution can indeed be stored for a long time.

Our study had several strengths. First, we included CDI patients in the gray area in the aspect of response to antibiotic treatment. As these patients form a large population in clinical practice, the evidence supporting FMT for partially treated patients provided in this study is very relevant. Second, we prospectively collected data on various factors that could influence the outcome of FMT; very few studies have reported results of FMT considering the Charlson comorbidity index. In addition, we performed microbiome analysis of donors and recipients. Third, all decisions were taken and FMT procedures performed by one physician. This reduced bias related to the FMT procedure. However, our study also had limitations. First, there is a possibility that we missed a meaningful factor affecting the outcome of FMT due to insufficient statistical power stemming from the small sample size. Second, we could not analyze metabolites in the stools. Therefore, we could not examine the recent hypothesis that bile acid and bile salt hydrolase- 
producing bacteria are related to the pathogenesis of $\mathrm{CDI}^{29}$

In conclusion, FMT is highly effective in partially treated CDI, with good short-term resolution of symptoms and long-term prevention of CDI recurrence. However, it should be considered prudently in refractory CDI because the outcome is not as excellent as in partially treated CDI.

\section{CONFLICTS OF INTEREST}

No potential conflict of interest relevant to this article was reported.

\section{ACKNOWLEDGEMENTS}

This study was supported by a grant (no. 14-2015-032) from the Seoul National University Bundang Hospital Research Fund and by the Bio \& Medical Technology Development program of the National Research Foundation of Korea (NRF), funded by the Ministry of Science and ICT (MIST) of the Republic of Korea (project no. 2016M3A9F3947027).

\section{AUTHOR CONTRIBUTIONS}

Study concept and enrolled the subjects: H.Y. Data collection: H.Y., H.I.S. Data analysis: H.Y. Microbial culture: M.S. Writing - original draft: H.Y. Writing - review \& editing: C.M.S., Y.S.P., N.K., D.H.L. All authors approved the final version of the manuscript.

\section{ORCID}

Hyuk Yoon Hyun Ik Shim Mijin Seol Cheol Min Shin Young Soo Park Nayoung Kim Dong Ho Lee https://orcid.org/0000-0002-2657-0349 https://orcid.org/0000-0001-5551-1180 https://orcid.org/0000-0001-6039-9787 https://orcid.org/0000-0003-2265-9845 https://orcid.org/0000-0003-1893-7726 https://orcid.org/0000-0002-9397-0406 https://orcid.org/0000-0002-6376-410X

\section{REFERENCES}

1. McDonald LC, Gerding DN, Johnson S, et al. Clinical practice guidelines for Clostridium difficile infection in adults and children: 2017 update by the Infectious Diseases Society of America (IDSA) and Society for Healthcare Epidemiology of America (SHEA). Clin Infect Dis 2018;66:987-994.

2. Song JH, Kim YS. Recurrent Clostridium difficile infection: risk factors, treatment, and prevention. Gut Liver 2019;13:16-24.

3. Quraishi MN, Widlak M, Bhala N, et al. Systematic review with meta-analysis: the efficacy of faecal microbiota transplantation for the treatment of recurrent and refractory Clostridium difficile infection. Aliment Pharmacol Ther 2017;46:479-493.

4. Mullish BH, Quraishi MN, Segal JP, et al. The use of faecal microbiota transplant as treatment for recurrent or refractory Clostridium difficile infection and other potential indications: joint British Society of Gastroenterology (BSG) and Healthcare Infection Society (HIS) guidelines. Gut 2018;67:1920-1941.

5. Surawicz CM, Brandt LJ, Binion DG, et al. Guidelines for diagnosis, treatment, and prevention of Clostridium difficile infections. Am J Gastroenterol 2013;108:478-498.

6. Kelly CR, Ihunnah C, Fischer M, et al. Fecal microbiota transplant for treatment of Clostridium difficile infection in immunocompromised patients. Am J Gastroenterol 2014;109:1065-1071.

7. Zainah H, Hassan M, Shiekh-Sroujieh L, Hassan S, Alangaden $G$, Ramesh M. Intestinal microbiota transplantation, a simple and effective treatment for severe and refractory Clostridium difficile infection. Dig Dis Sci 2015;60:181185.

8. Burke KE, Lamont JT. Clostridium difficile infection: a worldwide disease. Gut Liver 2014;8:1-6.

9. Cammarota G, Ianiro G, Tilg H, et al. European consensus conference on faecal microbiota transplantation in clinical practice. Gut 2017;66:569-580.

10. Gweon TG, Kim J, Lim CH, et al. Fecal microbiota transplantation using upper gastrointestinal tract for the treatment of refractory or severe complicated Clostridium difficile infection in elderly patients in poor medical condition: the first study in an Asian country. Gastroenterol Res Pract 2016;2016:2687605.

11. Cohen NA, Livovsky DM, Yaakobovitch S, et al. A retrospective comparison of fecal microbial transplantation methods for recurrent Clostridium difficile infection. Isr Med Assoc J 2016;18:594-599.

12. Chun J, Kim KY, Lee JH, Choi Y. The analysis of oral microbial communities of wild-type and toll-like receptor 2-deficient mice using a 454 GS FLX Titanium pyrosequencer. BMC Microbiol 2010;10:101.

13. Hur M, Kim Y, Song HR, Kim JM, Choi YI, Yi H. Effect of genetically modified poplars on soil microbial communities during the phytoremediation of waste mine tailings. Appl Environ Microbiol 2011;77:7611-7619.

14. Kim BS, Kim JN, Yoon SH, Chun J, Cerniglia CE. Impact of 
enrofloxacin on the human intestinal microbiota revealed by comparative molecular analysis. Anaerobe 2012;18:310-320.

15. Yoon SH, Ha SM, Kwon S, et al. Introducing EzBioCloud: a taxonomically united database of $16 \mathrm{~S}$ rRNA gene sequences and whole-genome assemblies. Int J Syst Evol Microbiol 2017;67:1613-1617.

16. Kelly CR, Khoruts A, Staley C, et al. Effect of fecal microbiota transplantation on recurrence in multiply recurrent Clostridium difficile infection: a randomized trial. Ann Intern Med 2016;165:609-616.

17. Kao D, Roach B, Silva M, et al. Effect of oral capsule- vs colonoscopy-delivered fecal microbiota transplantation on recurrent Clostridium difficile infection: a randomized clinical trial. JAMA 2017;318:1985-1993.

18. van Nood E, Vrieze A, Nieuwdorp M, et al. Duodenal infusion of donor feces for recurrent Clostridium difficile. $\mathrm{N}$ Engl J Med 2013;368:407-415.

19. Cammarota G, Masucci L, Ianiro G, et al. Randomised clinical trial: faecal microbiota transplantation by colonoscopy vs. vancomycin for the treatment of recurrent Clostridium difficile infection. Aliment Pharmacol Ther 2015;41:835843.

20. Hota SS, Sales V, Tomlinson G, et al. Oral vancomycin followed by fecal transplantation versus tapering oral vancomycin treatment for recurrent Clostridium difficile infection: an open-label, randomized controlled trial. Clin Infect Dis 2017;64:265-271.

21. Feuerstadt P, Das R, Brandt LJ. The evolution of urban C. difficile infection (CDI): CDI in 2009-2011 is less severe and has better outcomes than CDI in 2006-2008. Am J Gastroenterol 2014;109:1265-1276.
22. Aroniadis OC, Brandt LJ, Greenberg A, et al. Long-term follow-up study of fecal microbiota transplantation for severe and/or complicated Clostridium difficile infection: a multicenter experience. J Clin Gastroenterol 2016;50:398-402.

23. Agrawal M, Aroniadis OC, Brandt LJ, et al. The long-term efficacy and safety of fecal microbiota transplant for recurrent, severe, and complicated Clostridium difficile infection in 146 elderly individuals. J Clin Gastroenterol 2016;50:403407.

24. Ooijevaar RE, van Beurden YH, Terveer EM, et al. Update of treatment algorithms for Clostridium difficile infection. Clin Microbiol Infect 2018;24:452-462.

25. Li X, Gao X, Hu H, et al. Clinical efficacy and microbiome changes following fecal microbiota transplantation in children with recurrent Clostridium difficile infection. Front Microbiol 2018;9:2622.

26. Seekatz AM, Aas J, Gessert CE, et al. Recovery of the gut microbiome following fecal microbiota transplantation. mBio 2014;5:e00893-14.

27. Costello SP, Conlon MA, Vuaran MS, Roberts-Thomson IC, Andrews JM. Faecal microbiota transplant for recurrent Clostridium difficile infection using long-term frozen stool is effective: clinical efficacy and bacterial viability data. Aliment Pharmacol Ther 2015;42:1011-1018.

28. Terveer EM, van Beurden YH, Goorhuis A, et al How to: establish and run a stool bank. Clin Microbiol Infect 2017;23:924-930.

29. Mullish BH, McDonald JAK, Pechlivanis A, et al. Microbial bile salt hydrolases mediate the efficacy of faecal microbiota transplant in the treatment of recurrent Clostridioides difficile infection. Gut 2019;68:1791-1800. 